\title{
Occupational noise exposure and the prevalence of dyslipidemia in a cross- sectional study
}

\author{
Kun Zhang ${ }^{1}$, Feng Jiang ${ }^{2}$, Haibin Luo ${ }^{1}$ and Fangwei Liu ${ }^{1 *}$ (D)
}

\begin{abstract}
Background: Occupational noise exposure was related to cardiovascular disease, of which dyslipidemia was an important inducement. This study investigated the relationship between occupational noise exposure and dyslipidemia.

Methods: Four hundred ninety-two occupational noise-exposed workers and 664 non-exposed workers were recruited to conduct environmental noise tests and personal occupational physical examinations. A lasso-logistic regression model was used to estimate the relative risk of dyslipidemia. A restricted cubic spline was used to estimate the association between noise exposure years and dyslipidemia after adjusting for potential confounding factors.

Results: A crude association was observed between the occupational noise exposure (75-85 dB(A)) and dyslipidemia. After adjusting for confounding factors, there was a non-linear relationship between noise exposure years and dyslipidemia ( $\mathrm{P}$ for non-linearity $=0.01$ ). Workers exposed to $75-85 \mathrm{~dB}(\mathrm{~A})$ for 11 to 24.5 years had a higher risk of dyslipidemia than non-exposed workers.

Conclusions: A positive and non-linear exposure-response relationship was found in workers exposed to 75-85 $\mathrm{dB}(\mathrm{A})$ whose exposure years were between 11 and 24.5. Workers had the highest risk of dyslipidemia when exposed for 13.5 years.
\end{abstract}

Keywords: Occupational noise exposure, Dyslipidemia, Lasso-logistic regression, Restricted cubic spline

\section{Background}

As an environmental and occupational pollutant, the detrimental effect of noise has attracted more and more attention [1,2]. Epidemiological studies have reported increased mortality and incidence of cardiovascular diseases after chronic occupational noise exposure [3-6]. Noise, a psychosocial stressor, could lead to the increase of blood lipids, blood pressure, and blood glucose, which were the pathological basis of cardiovascular disease [7].

\footnotetext{
* Correspondence: fwliu@cmu.edu.cn

${ }^{1}$ Division of Pneumoconiosis, School of Public Health, China Medical University, No.77 Puhe Road, Shenyang North New Area, Shenyang 110122, People's Republic of China

Full list of author information is available at the end of the article
}

The possible biological mechanism was that noise impaired the hormones and metabolic regulation by activating the sympathetic nerve and endocrine system $[8$, 9]. Therefore, studying the effects of noise on blood lipids, blood pressure and blood glucose was vital for the prevention of cardiovascular disease.

At present, a large amount of evidences show that noise exposure could cause an increase in blood pressure and blood glucose [10-13]. However, there were relatively few studies on the relationship between noise and blood lipids. Some researchers have observed that there were increased cholesterol and triglyceride when workers were exposed to occupational noise above 80

(c) The Author(s). 2021 Open Access This article is licensed under a Creative Commons Attribution 4.0 International License, which permits use, sharing, adaptation, distribution and reproduction in any medium or format, as long as you give appropriate credit to the original author(s) and the source, provide a link to the Creative Commons licence, and indicate if changes were made. The images or other third party material in this article are included in the article's Creative Commons licence, unless indicated otherwise in a credit line to the material. If material is not included in the article's Creative Commons licence and your intended use is not permitted by statutory regulation or exceeds the permitted use, you will need to obtain permission directly from the copyright holder. To view a copy of this licence, visit http://creativecommons.org/licenses/by/4.0/ The Creative Commons Public Domain Dedication waiver (http://creativecommons.org/publicdomain/zero/1.0/) applies to the data made available in this article, unless otherwise stated in a credit line to the data. 
$\mathrm{dB}(\mathrm{A})[14,15]$. Nevertheless, dyslipidemia has not been confirmed by others $[4,16,17]$. A study manifested no association between occupational noise exposure and dyslipidemia, although adjusted for 11 confounding factors such as sex, age, and body mass index (BMI) [18]. In addition, the effect of noise on blood lipids needed to be accumulated over a long period of time, but few studies explored the relationship between noise exposure years and blood lipids.

Nowadays, The least absolute shrinkage and selection operator (lasso)-logistic regression and restricted cubic spline (RCS) have been used to analyze the relationship between disease and risk factors $[19,20]$. Lasso-logistic regression was a combination of lasso regression and logistic regression. When the regression model had plentiful covariates, the stepwise selection approach was not optimal, while lasso regression had many desirable properties [21, 22]. Lasso regression has been proven to effectively eliminate multicollinearity among confounding factors [23]. Therefore it could screen out the factors which had a direct impact on the dependent variable among these confounding factors. The restricted cubic spline could detect the possible nonlinear relationship between the dependent variable and the main independent variable through a continuous curve [24]. While the two methods have not been applied to screening and analyzing the relationship between occupational noise exposure and dyslipidemia.

The purpose of this study was to use a more advantageous model to screen confounding variables and investigate the exposure-response relationship between occupational noise exposure and the risk of dyslipidemia.

\section{Methods}

\section{Study population}

Two aviation manufacturing plants were randomly selected from all aviation manufacturing companies in Shenyang. In the two plants, all workers working in noise exposure workplaces were selected as the exposure group. The non-exposed group was included the workers who had no specific noise exposure in the workplace in the same plant and met the hygienic standard for noise in industrial enterprises (GB 3096-2008). A total of 1513 workers were included.

The inclusion criteria were workers without a family history of deafness, dyslipidemia, or other cardiovascular diseases; history of head trauma, blast exposure, hearing system disease, major liver disease, and ototoxic drug; and missing data. According to the Occupational Health Standard of the People's Republic of China: Measurement of Noise in the Workplace (GBZ/T 189.8-2007) (China, 2007), the Chinese workplace noise exposure limit was $85 \mathrm{~dB}(\mathrm{~A})$, and this study aimed to explore the impact of noise below it on blood lipids. What's more, some studies which also considered the impact of lowerlever noise exposure on non-auditory systems chose 75 $\mathrm{dB}(\mathrm{A})$ as the cut-off value $[18,25,26]$. Therefore, the workers with occupational noise exposure had a duration of more than 1 year and the intensity of noise exposure was $75-85 \mathrm{~dB}$ (A) (LEX,8 h).

Each participant performed an occupational health examination in 2018, which included a history and physical examination (Additional file 1, was developed for this study). In the exposed group, noise exposure years were recorded, which was the working years of workers who were still working in the noise exposure workshop and have not been transferred by the physical examination. Professional physicians determined the height, weight, heart rate, electrocardiogram, blood pressure, blood glucose, blood lipids, fatty liver, and hearing impairment. Body mass index (BMI) was calculated by dividing body weight $(\mathrm{kg})$ by the square of height $(\mathrm{m})$.

Finally, 492 occupational noise-exposed participants (exposed group) and 664 non-noise-exposed participants (non-exposed group) were included in this crosssectional study.

\section{Definition of dyslipidemia}

Blood tests were performed to assess triglyceride (TG), serum total cholesterol (TC), high-density lipoprotein cholesterol (HDL-C), and low-density lipoprotein cholesterol (LDL-C). The judgment of dyslipidemia referred to the standards of the "Guidelines for the Prevention and Treatment of Dyslipidemia in Adults in China (2016 Revised Edition)": (1) TC $\geq 6.2 \mathrm{mmol} / \mathrm{L}$; (2) HDL-C $<1.0$ $\mathrm{mmol} / \mathrm{L}$; (3) $\mathrm{LDL}-\mathrm{C} \geq 4.1 \mathrm{mmol} / \mathrm{L}$; (4) $\mathrm{TG} \geq 2.3 \mathrm{mmol} / \mathrm{L}$. Dyslipidemia was defined as the presence of one or more of these components.

\section{Assessment of noise exposure}

Noise intensity in the workplace was determined by a noise statistical analyzer (HS6288E). According to the occupational health standard in China (GBZ/T 189.82007), noise exposure was evaluated with equivalent continuous $\mathrm{dB}(\mathrm{A})$-weighted sound pressure levels (LEX, $8 \mathrm{~h})$. Based on the occupational noise exposure assessment, the intensity of noise in the workplace was $75-85$ $\mathrm{dB}(\mathrm{A})$ (LEX,8 h).

\section{Assessment of pure-tone audiometry}

The degree of hearing impairment was identified by pure-tone audiometry. Based on the National Occupational Health Standard of the People's Republic of China, an audiometer (AD28) was used to measure the air conduction hearing thresholds at the speech frequen$\operatorname{cies}(500,1000,2000 \mathrm{~Hz})$ and the high frequencies $(3000$, $4000,6000 \mathrm{~Hz})$. The hearing thresholds for these 
frequencies $26 \mathrm{~dB}(\mathrm{~A})$ or higher in any ear were defined as the increased hearing threshold.

\section{Statistical analysis}

SAS (university edition) was used to analyze descriptive statistics between the noise exposure group and the non-noise exposure group. The Shapiro-Wilk test was used to assess the normality of continuous variables. Because continuous variables distributed abnormally, summary values were median (P25, P75). Categorical variables were expressed as frequencies (\%). Sorted all variables in layers, the $x^{2}$ test was used to compare the differences between groups.

Tibshirani proposed a penalty regression methodlasso in 1996, which was characterized by variable selection and complexity adjustment while fitting a generalized linear model [23]. Regardless of the nature of the dependent variable, lasso regression could be used for modeling.

The basic idea of lasso regression was that each regression coefficient was compressed by a penalty function, and even some regression coefficients became 0 , so that a simplified model with fewer variables could be obtained. The logistic regression model established by the lasso method for variable selection was the lasso-logistic regression model.

This study discussed the effect of noise exposure on blood lipids, which was a binary model problem. According to the conditions of dyslipidemia prevalence, all participants were divided into non-diseased and diseased, where 0 meant non-diseased and 1 meant diseased. Since the conditions of dyslipidemia prevalence were independent of each other, let $\left(\mathrm{X}_{\mathrm{i}}, \mathrm{Y}_{\mathrm{i}}\right)$ be the sample value of the dyslipidemia condition of $n$ workers. $X_{i}$ was the observed value of $p$ different attributes of the $i$ th worker. $Y_{i}$ was the dependent variable, and $Y_{i} \in\{0,1\}$. The probability of a worker suffering from dyslipidemia was assumed $\pi_{\mathrm{i}}=\mathrm{p}\left(\mathrm{Y}_{\mathrm{i}}=1 \mid \mathrm{X}_{\mathrm{i}}\right)$. $\beta$ was the logistic regression coefficient, and $\lambda p(\beta)$ was the penalty term (1).

$$
\lambda p(\beta)=\lambda \sum_{j=1}^{p}\left|\beta_{j}\right|
$$

Then the estimated coefficient $\hat{\beta}$ in the lasso-logistic regression model could be written in the form of the formula (2).

$$
\begin{aligned}
\hat{\beta}= & \arg \min \left[-\sum\left\{Y_{i} \ln \left(\pi_{i}\right)+\left(1-Y_{i}\right) \ln \left(1-\pi_{i}\right)\right\}\right] \\
& +\lambda p(\beta)
\end{aligned}
$$

The first half of the formula (2) expressed the degree of model fitting, and the second half expressed the punishment for variables. $\lambda(\lambda \geq 0)$ was used as a penalty parameter to determine the degree of compression of model coefficients. The penalty function was to punish the absolute value of the regression coefficients, requiring the sum of the absolute values of all regression coefficients to be less than or equal to the penalty parameter $\lambda$. According to the requirements of penalty conditions, $\sum_{j=1}^{p}\left|\beta_{j}\right| \leq \lambda$. As $\lambda$ increased, the coefficients of each variable were gradually compressed. When $\lambda \rightarrow \infty$, all coefficients were compressed to zero.

In the lasso model, the size of the penalty parameter $\lambda$ was directly related to the complexity and convergence of the final model. Therefore, choosing a reasonable and scientific parameter tuning method was of great significance to the final effect of the lasso model. The simplest and most widely used method to select the appropriate value of $\lambda$ was the cross-validation method [27]. In this method, the samples were divided into $\mathrm{n}$ sub-samples, and the $n$ groups of sub-samples were cross-validated $n$ times to obtain $\mathrm{n}$ times the model fitting situation, from which the optimal $\lambda$ was selected.

Therefore, the Lasso regression model was used to screen the risk factors of dyslipidemia and indicated the degree of importance of all factors. The cross-validation method was used to calculate $\lambda$ value with the smallest error, which corresponded to the screened risk factors. A binary logistic regression model was used to assess the association between noise exposure status and dyslipidemia, with adjusting for the other screened factors. The results were presented as odds ratio (OR) with their 95\% confidence intervals (CI). A restricted cubic spline was used to flexibly model the association of duration of noise exposure (year) and dyslipidemia prevalence. In the spline model, other screened factors were adjusted. The non-linearity assumption was tested by using a likelihood ratio test.

In addition, a $p$-value of $<0.05$ was used to define statistical significance in all analyses. Apart from descriptive statistics, other statistical analyses were conducted in $\mathrm{R}$ (version 3.6.2).

\section{Results}

In total, 1513 workers were included in the study. The baseline characteristics of participants in both the exposed group and the non-exposed group were shown in Table 1, of whom most were focused on the age of 3039. The population of the study included more males than females in both groups. Significant differences were identified between the exposed group and the nonexposed group in sex, BMI, PTA, hypertension, fatter liver, and diabetes (all P's $<0.05$ ). The median of noise exposure years was $13(8,19)$ years, with a noise intensity value of $78.90(78.10,82.10) \mathrm{dB}(\mathrm{A})$. 
Table 1 Demographic Characteristics of Employees by Workplace Noise Exposure

\begin{tabular}{|c|c|c|c|c|c|c|c|}
\hline \multirow[t]{2}{*}{ Variables } & \multicolumn{3}{|c|}{ Noise-exposed group } & \multicolumn{3}{|c|}{ control group } & \multirow{2}{*}{$\begin{array}{l}P \\
\text { value }^{\mathrm{a}}\end{array}$} \\
\hline & No. & $\%$ & M (QL, QU) & No. & $\%$ & M (QL, QU) & \\
\hline Age & & & $35(29,39)$ & & & $34(30,39)$ & 0.300 \\
\hline$<30$ & 136 & 27.64 & & 160 & 24.10 & & \\
\hline $30-39$ & 251 & 51.02 & & 376 & 56.63 & & \\
\hline $40-49$ & 67 & 13.62 & & 80 & 12.05 & & \\
\hline $50-59$ & 38 & 7.72 & & 48 & 7.23 & & \\
\hline Sex & & & & & & & $<0.001$ \\
\hline Male & 461 & 93.70 & & 471 & 70.93 & & \\
\hline Female & 31 & 6.30 & & 193 & 29.07 & & \\
\hline Heart Rate & & & $77(71,85)$ & & & $79(72,86)$ & 0.259 \\
\hline$<60$ & 12 & 2.44 & & 8 & 1.20 & & \\
\hline $60-100$ & 466 & 94.72 & & 634 & 95.48 & & \\
\hline$>100$ & 14 & 2.85 & & 22 & 3.31 & & \\
\hline BMI & & & $25.29(22.66,27.96)$ & & & $23.73(21.22,26.20)$ & $<0.001$ \\
\hline$<18.5$ & 12 & 2.44 & & 28 & 4.22 & & \\
\hline $18.5-23.9$ & 161 & 32.72 & & 325 & 48.95 & & \\
\hline $24-27.9$ & 200 & 40.65 & & 242 & 36.45 & & \\
\hline$\geq 28$ & 119 & 24.19 & & 69 & 10.39 & & \\
\hline ECG & & & & & & & 0.254 \\
\hline Normal & 362 & 73.58 & & 508 & 76.51 & & \\
\hline Abnormal & 130 & 26.42 & & 156 & 23.49 & & \\
\hline PTA & & & & & & & $<0.001$ \\
\hline Normal & 465 & 94.51 & & 664 & 100.00 & & \\
\hline Abnormal & 27 & 5.49 & & 0 & 0.00 & & \\
\hline Hypertension & & & & & & & $<0.001$ \\
\hline Yes & 210 & 42.68 & & 188 & 28.31 & & \\
\hline No & 282 & 57.32 & & 476 & 71.69 & & \\
\hline Fatty liver & & & & & & & $<0.001$ \\
\hline Yes & 279 & 56.71 & & 243 & 36.60 & & \\
\hline No & 213 & 43.29 & & 421 & 63.40 & & \\
\hline Diabetes & & & & & & & 0.008 \\
\hline Yes & 22 & 4.47 & & 12 & 1.81 & & \\
\hline No & 470 & 95.53 & & 652 & 98.19 & & \\
\hline Noise exposure years (years) & & & $13(8,19)$ & & & & \\
\hline Noise intensity $(\mathrm{dB}(\mathrm{A}))$ & & & $78.90(78.10,82.10)$ & & & & \\
\hline
\end{tabular}

Abbreviations: $B M I$ body mass index, ECG electrocardiogram, PTA pure-tone audiometry

${ }^{a} X^{2}$ test for the difference $(P<0.05)$ between the 2 groups

The median levels of TG, TC, HDL, and LDL and the odds ratio of dyslipidemia between the two groups were presented in Table 2. Compared with the non-exposed group, the exposed group had significantly higher levels in TG, TC, and LDL $(P<0.001)$. Workers exposed to noise had a higher odds ratio of dyslipidemia (unadjusted odds ratio $=1.81,95 \% \mathrm{CI}: 1.40,2.35$ ).

After baseline description, age, sex, heart rate, BMI, ECG, PTA, hypertension, fatty liver, and diabetes were incorporated into the Lasso regression model, which was used to screen variables. $\operatorname{Lambda}(\lambda)$ value was shown by the cross-validation method in Fig. 1(a). The range between the two dashed lines indicated the range of positive and negative standard deviations of the lambda value. Within the range, the deviation of the regression model fluctuated slightly. Thus, the lambda value with the smallest mean-squared error in the range was chosen, which included 7 variables. The coefficient of each risk factor was compressed as the lambda value became larger (Fig. 1(b)). The earlier the variable was compressed, the less important it was. Ultimately the top seven risk factors, fatter liver, 
Table 2 Rest of blood lipids and odds ratio of Dyslipidemia by Noise Group, Shenyang, Liaoning,2018

\begin{tabular}{|c|c|c|c|c|c|c|c|c|}
\hline \multirow[t]{2}{*}{ Noise Group } & \multicolumn{4}{|c|}{$\begin{array}{l}\text { Median (QL; QU) } \\
\text { Blood lipids (mmol/L) }\end{array}$} & \multirow{2}{*}{$\begin{array}{l}\text { No. of dyslipidemsia } \\
\text { Cases }\end{array}$} & \multicolumn{3}{|c|}{ Risk of Dyslipidemia (Unadjusted) } \\
\hline & TG & TC & HDL & LDL & & $\overline{O R}$ & $95 \% \mathrm{Cl}$ & $P$ Value $^{\mathrm{b}}$ \\
\hline Control & $1.13(0.73 ; 1.81)$ & $4.74(4.21 ; 5.41)$ & $1.43(1.22 ; 1.71)$ & $2.60(2.20 ; 3.10)$ & 153 & 1.00 & Referent & \\
\hline Noise-exposed & $1.44(0.99 ; 2.43)$ & $4.89(4.25 ; 5.56)$ & $1.30(1.18 ; 1.46)$ & $2.82(2.33 ; 3.30)$ & 173 & 1.81 & $(1.40,2.35)$ & $<0.001$ \\
\hline$P$ Value ${ }^{a}$ & $<0.001$ & $<0.001$ & $<0.001$ & $<0.001$ & & & & \\
\hline
\end{tabular}

Abbreviation: TG triglyceride, TC serum total cholesterol, $H D L$ high-density lipoprotein, $L D L$ low-density lipoprotein, $O R$ odds ratio, $C l$ confidence interval

a Wilcoxon two-sample test for the difference between the two groups

b Simple logistic regression model

BMI, hypertension, diabetes, sex, noise exposure, and age, were retained.

Thus, the 7 selected variables were included for logistic regression analysis. The association between noise exposure and prevalence of dyslipidemia was shown in Fig. 2. After adjusting for fatty liver, diabetes, hypertension, sex, age, and BMI, no significant difference was found in the relationship between occupational noise exposure and prevalence of dyslipidemia (P's $>0.05$ ). Workers with a BMI of 24-27.9 had a higher relative risk of dyslipidemia than workers with a BMI of 18.523.9, and the strongest association was observed at the BMI of $\geq 28$. Participants with fatty liver, diabetes, hypertension, and male had an increased probability of having dyslipidemia, respectively.

Further, the exposure-response relationship between the continuity of noise exposure and dyslipidemia is worthy of further research. Therefore, the noise exposure years were introduced to explore the effect of longterm exposure on dyslipidemia. A restricted cubic spline was used to observe the relation of noise exposure years and prevalence of dyslipidemia in Fig. 3. After adjusting for fatty liver, diabetes, hypertension, sex, age, and BMI, the risk of prevalent dyslipidemia was not significantly correlated with noise exposure years until around 11 years and then increased during 11-13.5 years and decreased above 13.5 years $(\mathrm{P}$ for non-linearity $=0.01$ ).

\section{Discussion}

\section{Summary}

The study explored the correlation between occupational noise exposure and the prevalence of dyslipidemia by modeling. First, well-established extraneous risk factors of blood lipid levels were accounted for and screened. Interestingly, the crude association between occupational noise exposure and dyslipidemia vanished after adjusting for the screened confounders. Second, we explored the long-term changes of chronic noise exposure to dyslipidemia. Our findings suggested there was a non-linear relationship between noise exposure years and dyslipidemia among workers in aviation manufacturing plants. Workers exposed to occupational noise (75-85
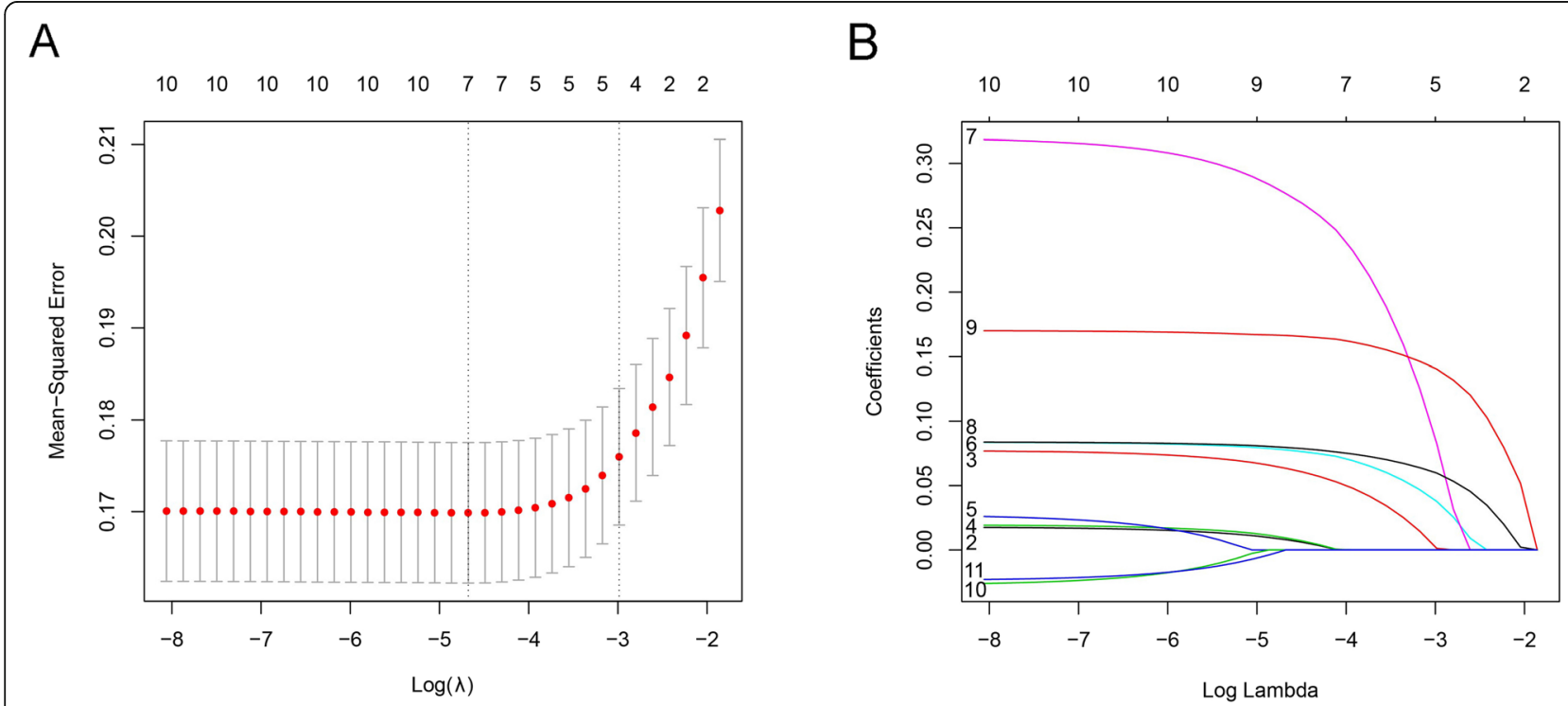

Fig. 1 The screening of variable. a The trend between lambda $(\lambda)$ and the number of variables. A cross-validation method was used. $\mathbf{b}$ The relationship between lambda $(\lambda)$ and lasso coefficients.2, Age;3, Sex;4, Noise exposure;5, PTA;6, Hypertension;7, Diabetes;8, BMl;9, Fatty liver;10, Heart rate; 11, EGC 


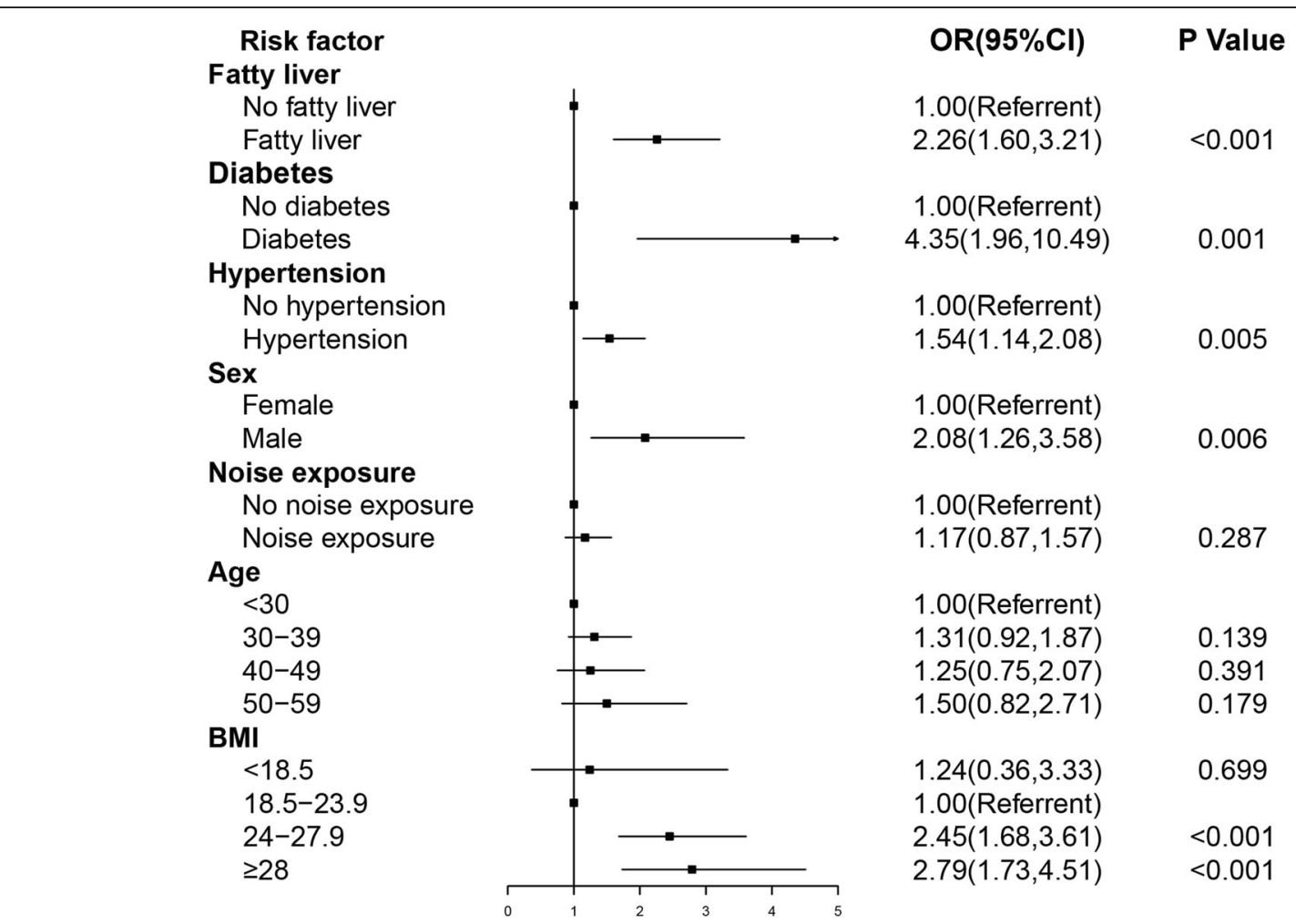

Fig. 2 Forest plot for performance on the odds ratio of prevalent dyslipidemia by exposing occupational noise. A logistic regression model was used. OR, odds ratio; BMl: body mass index; Cl, confidence interval

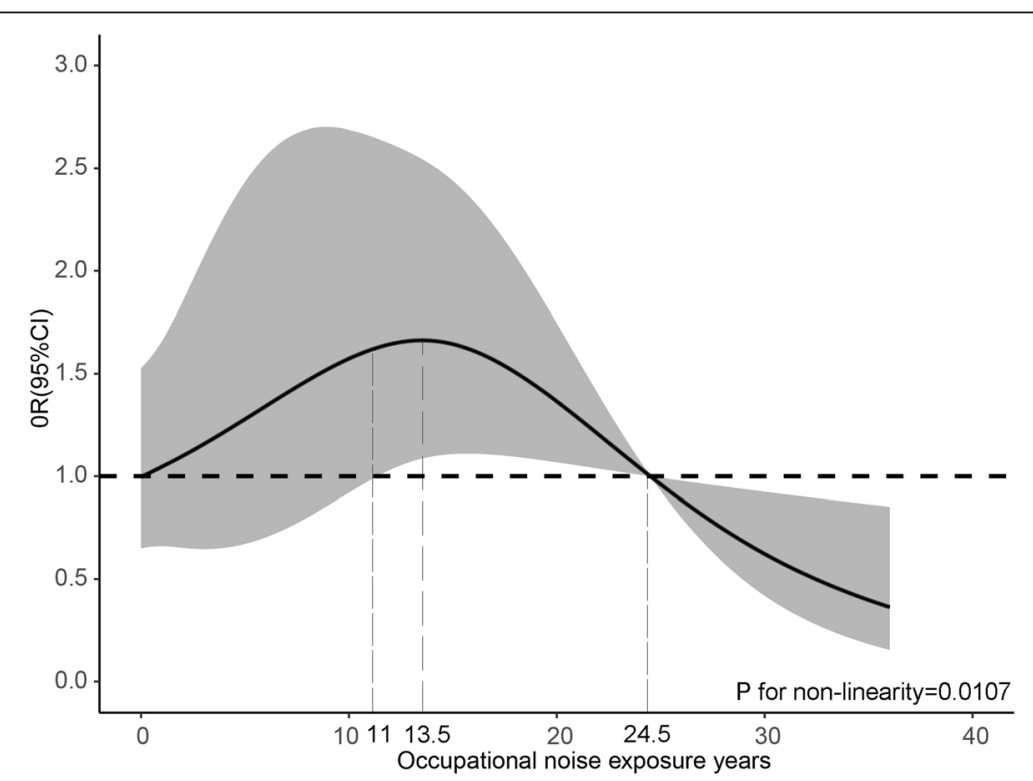

Fig. 3 Association (odds ratio) between occupational noise exposure years and dyslipidemia prevalence among 1156 participants. A restricted cubic spline with 4 knots was modeled. Analysis was adjusted for fatty liver, diabetes, hypertension, sex, age, and BMI.OR, odds ratio; Cl, confidence interval 
$\mathrm{dB}(\mathrm{A}))$ for 11-24.5 years had a higher prevalence of dyslipidemia than those without noise exposure.

\section{Lasso-logistic regression---screening and linear analysis of risk factors of dyslipidemia}

Epidemiological evidence has demonstrated that longterm exposure to noise increased the risk of cardiovascular disease $[2,28]$. Occupational noise-induced dyslipidemia might be an important intermediate link in subsequent major cardiovascular events. But, the actual relationship between occupational noise exposure and dyslipidemia was still under investigation. Their association was observed in young men $(\leq 44 \mathrm{y})$ after adjusting for a limited set of extraneous risk factors [14]. Another study also showed a significantly different level of TG between noise-exposed workers and non-exposed workers after adjustment for some confounders [29]. On the contrary, some researchers demonstrated that there was no association between noise exposure level and blood lipid levels $[4,16,17]$. Therefore, novel perspectives and appropriate methods were needed to explore the real relationship between occupational noise and dyslipidemia.

In this study, ten recognized risk variables, noise exposure, age, sex, heart rate, BMI, ECG, PTA, hypertension, fatty liver, and diabetes were included to explore the association between occupational noise and dyslipidemia. The relationships among these risk factors were complicated and had a certain correlation. Existing studies about the impact of occupational noise exposure on dyslipidemia were mostly using stepwise logistic regression to screen variables. However, the results obtained by using this method were inherently discrete and unstable so that it was difficult to screen the most meaningful variables [30, 31]. Our study used Lasso regression to screen out seven variables: noise exposure, age, sex, hypertension, fatty liver, diabetes, and BMI. Because lasso regression had the advantages of strong predictive ability and high stability. What's more, it retained a few significant variables and eliminated the influence of multicollinearity by reducing the insignificant coefficient to 0 [23]. After that, the screened variables were included in the logistic regression model analysis. The processes of screening and analyzing variables were called lasso-logistic regression. Our results have shown that there was no association between occupational noise exposure and dyslipidemia when other confounding factors were considered, which were consistent with others [18]. The reason might be that the noise intensity in the study was weaker than the national exposure limit of $85 \mathrm{~dB}(\mathrm{~A})$ (LEX, $8 \mathrm{~h}$ ), and low-level noise might have less impact on human physiology. A study found a similar result that when the noise intensity was below 85 $\mathrm{dB}(\mathrm{A})$ (LEX,8 h), no significant difference was found in the risk of hypertension between noise-exposed and non-exposed groups [32].

\section{The exposed-response relationship between noise exposure years and dyslipidemia}

Existing theories believed that the changes in blood pressure were unstable in the early stage of noise exposure. And long-term exposure to high-intensity noise could lead to a continuous increase in blood pressure $[33,34]$. Similarly, the early effect of noise exposure on blood lipids was also not significant [35]. Moreover, some researches focused on the relationship between the intensity of noise and dyslipidemia, hypertension, and other diseases $[26,36,37]$. Our study included the continuous index of noise exposure years in order to observe the effect of long-term noise exposure on blood lipids. Because of the unknown relationship between noise exposure years and dyslipidemia, blindly using segmented regression could unify the internal effects of each segment. Importantly, it was difficult to find the maximum and minimum points in this method, which were obvious shortcomings in observing the exposedresponse relationship. In order to avoid the influence of model selection and impertinent grouping of noise exposure years, a restricted cubic spline combined with a logistic linear regression model was used to explore the relationship between noise exposure years and dyslipidemia. By this method, the effect of a small change in the independent variable was visualized with a continuous curve [24].

This study has shown that there was a non-linear exposed-response relationship between noise exposure years and dyslipidemia after adjusting for confounding factors. With the increase of noise exposure years, the risk of dyslipidemia presented a trend of rising first then falling. When the noise exposure years were between 11 and 13.5, the risk of dyslipidemia gradually increased, which indicated that the risk of dyslipidemia increased with the accumulation of noise exposure time. The risk of disease gradually decreased after 13.5 years of exposure. The reason might be that due to the physical deterioration or the occurrence of chronic diseases, some of the workers exposed to noise were transferred from their original positions, which gradually reduced the risk of dyslipidemia. Another study shown younger workers exposed to noise $(>80 \mathrm{~dB}(\mathrm{~A}))$ demonstrated a significant increase in TG, TC, and TC/HDL ratio, which confirmed our suspicions [14].

\section{Limitations}

To analyze the relationship between noise exposure and dyslipidemia more comprehensively, some factors can be considered in the future. Workers who have been exposed to noise by being transferred from the noise 
exposure position were not included, because they might have more complicated confounders that affected their blood lipids during the physical examination, such as the nature of new jobs and the resulting change in living and eating habits. What's more, the risk factors of dyslipidemia are complex, thereby some factors are still worth being explored, such as protective measures, living and eating habits, and genetic factors. In addition, participants' exposure to noise intensity outside of the workplace might not be measured. Furthermore, this study is a cross-sectional study, so causality could not be determined.

\section{Prediction and prevention of dyslipidemia}

In this study, the prediction model formed by lassologistic regression and restricted cubic spline had good prediction performance. The obtained exposureresponse model could be used as a tool for predicting dyslipidemia for workers exposed to noise. The model showed that when the noise intensity was $75-85 \mathrm{~dB}(\mathrm{~A})$ and the noise exposure years were 11-24.5, the prevalence of dyslipidemia increased.

On the one hand, according to the occupational health standard in China (GBZ/T 189.8-2007), the limit value for the prevention of auditory effect of occupational exposure to noise in China is $85 \mathrm{~dB}(\mathrm{~A})$. Although this limit was often used to discuss non-auditory effects [3], there was still a lack of limit values for the non-auditory effect of occupational noise exposure. Few studies used 75 $\mathrm{dB}(\mathrm{A})$ as the cut-off level. A study that only included workers exposed to noise found that when workers exposed to less than $75 \mathrm{~dB}(\mathrm{~A})$ were used as a reference, workers exposed to $75-85 \mathrm{~dB}(\mathrm{~A})$ were associated with hypertension [25]. Further, compared with non-noiseexposed workers in our study, with using more appropriate methods for modeling, workers exposed to 75-85 $\mathrm{dB}(\mathrm{A})$ of noise for a long time would still cause nonauditory effects such as dyslipidemia. Therefore, even if the noise was below the current limit of $85 \mathrm{~dB}(\mathrm{~A})$, the risk of non-auditory diseases would still increase. This finding may provide a theoretical basis for establishing Chinese limit values for the prevention of non-auditory effects of occupational noise exposure.

On the other hand, enterprises are supposed to strengthen regular monitoring and early intervention of risk factors, especially for workers with more than noise exposure years at 11 . It is vital to pay attention to the implementation of noise prevention and control measures and strengthen the personal protection of noiseexposed workers. Equally important, it is recommended to focus on providing nutrition intervention and health education to workers suffering from overweight and obesity, hypertension, diabetes, and fatty liver. Moreover, workers who have developed dyslipidemia can transfer from exposed positions in time to avoid aggravation or development of cardiovascular disease.

\section{Conclusions}

In summary, occupational noise exposure may be associated with an increased prevalence of dyslipidemia. Our study found a positive and non-linear exposure-response relationship was found in workers exposed to 75-85 $\mathrm{dB}(\mathrm{A})$ whose noise exposure years were between 11 and 24.5. Workers had the highest risk of dyslipidemia when exposed for 13.5 years.

\begin{abstract}
Abbreviations
BMl: Body mass index; Lasso: Least absolute shrinkage and selection operator; RCS: Restricted cubic spline; TG: Triglyceride; TC: Serum total Cholesterol; HDL-C: High-density lipoprotein cholesterol; LDL-C: Lowdensity lipoprotein cholesterol; OR: Odds ratio; Cl: Confidence intervals; ECG: Electrocardiogram; PTA: Pure-tone audiometry
\end{abstract}

\section{Supplementary Information}

The online version contains supplementary material available at https://doi. org/10.1186/s12889-021-11274-x.

Additional file 1. Occupational Health Examination Form (history examination section). This questionnaire collected the basic information, work position, occupational history, past disease history, family disease history, occupational disease history, menstrual history, reproductive history, smoking and drinking history, and symptom inquiry during examination.

\section{Acknowledgements}

We express our gratitude to Dr. Xiao Luo for her kind guide and suggestions in statistics.

\section{Authors' contributions}

FL and KZ conceptualized this analysis. FJ provided the resources of data. KZ and $\mathrm{HL}$ led the investigation of this study. KZ conducted the analysis and prepared the draft manuscript. FL revised the manuscript. All authors read and approved the final manuscript.

\section{Funding}

The work was supported by the Dengfeng Scholar of China Medical University (Grant No.1210618036).

\section{Availability of data and materials}

The datasets used and analysed during the current study are available from the corresponding author on reasonable request.

\section{Declarations}

Ethics approval and consent to participate

The study protocol was approved by the Medical Ethics Committee of China Medical University (permit number CMU 6204-3008). And all participants gave written informed consent prior to the survey and examination.

Consent for publication

Not applicable.

\section{Competing interests}

The authors declare that they have no competing interests.

\section{Author details}

'Division of Pneumoconiosis, School of Public Health, China Medical University, No.77 Puhe Road, Shenyang North New Area, Shenyang 110122, People's Republic of China. ${ }^{2}$ Health Management Center, Shenyang 242 Hospital, Shenyang, People's Republic of China. 
Received: 6 January 2021 Accepted: 11 June 2021

Published online: 29 June 2021

\section{References}

1. Schmidt F, Kolle K, Kreuder K, Schnorbus B, Wild P, Hechtner M, et al. Nighttime aircraft noise impairs endothelial function and increases blood pressure in patients with or at high risk for coronary artery disease. Clin Res Cardiol. 2015;104(1):23-30. https://doi.org/10.1007/s00392-014-0751-x.

2. Munzel T, Gori T, Babisch W, Basner M. Cardiovascular effects of environmental noise exposure. Eur Heart J. 2014;35(13):829-36. https://doi. org/10.1093/eurheartj/ehu030.

3. McNamee R, Burgess G, Dippnall WM, Cherry N. Occupational noise exposure and ischaemic heart disease mortality. Occup Environ Med. 2006; 63:813-9. https://doi.org/10.1136/oem.2005.026245

4. Virkkunen $\mathrm{H}$, Harma M, Kauppinen $\mathrm{T}$, Tenkanen L. The triad of shift work, occupational noise, and physical workload and risk of coronary heart disease. Occup Environ Med. 2006;63:378-86. https://doi.org/10.1136/oem.2 005.022558 .

5. Bortkiewicz A, Gadzicka E, Siedlecka J, Szyjkowska A, Viebig P, Wranicz JK, et al. Work-related risk factors of myocardial infarction. Int J Occup Med Environ Health. 2010;23:255-65. https://doi.org/10.2478/v10001-010-0030-7.

6. Chang T-Y, Hwang B-F, Liu C-S, Chen R-Y, Wang V-S, Bao B-Y, et al. Occupational noise exposure and incident hypertension in men: a prospective cohort study. Am J Epidemiol. 2013;177(8):818-25. https://doi. org/10.1093/aje/kws300.

7. Li W, Shu S, Cheng L, Hao X, Wang L, Wu Y, et al. Fasting serum total bile acid level is associated with coronary artery disease, myocardial infarction and severity of coronary lesions. Atherosclerosis. 2019;292:193-200. https:// doi.org/10.1016/j.atherosclerosis.2019.11.026.

8. Schmidt FP, Basner M, Kroeger G, Weck S, Schnorbus B, Muttray A, et al. Effect of nighttime aircraft noise exposure on endothelial function and stress hormone release in healthy adults. Eur Heart J. 2013;34:3508. https:// doi.org/10.1093/eurheartj/eht269.

9. Babisch W. The noise/stress concept, risk assessment and research needs. Noise Health. 2002;4(16):1-11.

10. Eze IC, Foraster M, Schaffner E, Vienneau D, Heritier H, Rudzik F, et al. Longterm exposure to transportation noise and air pollution in relation to incident diabetes in the SAPALDIA study. Int J Epidemiol. 2017:46(4):111525. https://doi.org/10.1093/ije/dyx020.

11. Roswall N, Raaschou-Nielsen O, Jensen SS, Tjonneland A, Sorensen M. Longterm exposure to residential railway and road traffic noise and risk for diabetes in a Danish cohort. Environ Res. 2018;160:292-7. https://doi.org/1 0.1016/j.envres.2017.10.008.

12. Brahem A, Riahi S, Chouchane A, Kacem I, Maalel OE, Maoua M, et al. Impact of occupational noise in the development of arterial hypertension: a survey carried out in a company of electricity production. Ann Cardiol Angeiol. 2019;68:168-74. https://doi.org/10.1016/j.ancard.2018.10.008.

13. Shaykhlislamova ER, Volgareva AD, Obukhova MP, Gimranova GG, Karimova LK, Valeeva ET. Prevalence of blood circulation diseases among workers exposed to occupational noise in mineral extraction and their workrelatedness. Siberian Sci Med J. 2018;38(6):137-44. https://doi.org/10.15372/ ssmj20180620.

14. Melamed S, Froom P, Kristal-Boneh E, Gofer D, Ribak J. Industrial noise exposure, noise annoyance, and serum lipid levels in blue-collar workers-the CORDIS study. Arch Environ Health. 1997;52(4):292-8. https://doi.org/1 0.1080/00039899709602201.

15. Vangelova KK, Deyanov CE. Blood pressure and serum lipids in industrial workers under intense noise and a hot environment. Rev Environ Health. 2007:22(4):303-11. https://doi.org/10.1515/reveh.2007.22.4.303.

16. Chang TY, Jain RM, Wain CS, Chan CC. Effects of occupational noise exposure on blood pressure. J Occup Environ Med. 2003;45(12):1289-96. https://doi.org/10.1097/01.jom.0000100003.59731.3d.

17. Virkkunen $H$, Kauppinen $T$, Tenkanen L. Long-term effect of occupational noise on the risk of coronary heart disease. Scand J Work Environ Health. 2005;31(4):291-9. https://doi.org/10.5271/sjweh.885.

18. Arlien-Soborg MC, Schmedes AS, Stokholm ZA, Grynderup MB, Bonde JP, Jensen CS, et al. Ambient and at-the-ear occupational noise exposure and serum lipid levels. Int Arch Occup Environ Health. 2016;89:1087-93. https:// doi.org/10.1007/s00420-016-1145-3.

19. Kim SM, Kim $Y$, Jeong $K$, Jeong $H$, Kim J. Logistic LASSO regression for the diagnosis of breast cancer using clinical demographic data and the BI-RADS lexicon for ultrasonography. Ultrasonography. 2018;37(1):36-42. https://doi. org/10.14366/usg.16045.

20. Lee DH, Keum N, Hu FB, Orav EJ, Rimm EB, Willett WC, et al. Predicted lean body mass, fat mass, and all cause and cause specific mortality in men: prospective US cohort study. Bmj. 2018;362:k2575. https://doi.org/10.1136/ bmj.k2575.

21. Houssami N, Irwig L, Simpson JM, McKessar M, Blome S, Noakes J. The influence of clinical information on the accuracy of diagnostic mammography. Breast Cancer Res Treat. 2004;85(3):223-8. https://doi.org/1 0.1023/b:Brea.0000025416.66632.84

22. Bickel PJ, Ritov Y, Tsybakov AB. Simultaneous analysis of lasso and dantzig selector. Ann Stat. 2009;37(4):1705-32. https://doi.org/10.1214/08-aos620.

23. Tibshirani R. Regression shrinkage and selection via the lasso; 1996.

24. Desquilbet $L$, Mariotti F. Dose-response analyses using restricted cubic spline functions in public health research. Stat Med. 2010;29:1037-57. https://doi.org/10.1002/sim.3841.

25. de Souza TC, Périssé AR, Moura M. Noise exposure and hypertension: investigation of a silent relationship. BMC Public Health. 2015;15:328. https:// doi.org/10.1186/s12889-015-1671-Z

26. Liu CS, Young LH, Yu TY, Bao BY, Chang TY. Occupational noise frequencies and the incidence of hypertension in a retrospective cohort study. Am J Epidemiol. 2016;184(2):120-8. https://doi.org/10.1093/aje/kwv333.

27. Hebiri M, Lederer J. How correlations influence lasso prediction. IEEE Trans Inf Theory. 2013;59(3):1846-54. https://doi.org/10.1109/tit.2012.2227680.

28. Babisch W. Cardiovascular effects of noise. Noise Health. 2011;13:201-4. https://doi.org/10.4103/1463-1741.80148.

29. Mehrdad R, Bahabad AM, Moghaddam AN. Relationship between exposure to industrial noise and serum lipid profile. Acta Med Iran. 2011;49(11):725-9.

30. Fan JQ, Li RZ. Variable selection via nonconcave penalized likelihood and its oracle properties. J Am Stat Assoc. 2001;96(456):1348-60. https://doi.org/1 $0.1198 / 016214501753382273$

31. Shen XT, Ye JM. Adaptive model selection. J Am Stat Assoc. 2002;97(457): 210-21. https://doi.org/10.1198/016214502753479356.

32. Chen $S, N i$, Z Zhang L, Kong L, Lu L, Yang Z, et al. Noise exposure in occupational setting associated with elevated blood pressure in China. BMC Public Health. 2017;17. https://doi.org/10.1186/s12889-017-4050-0.

33. Nserat S, Al-Musa A, Khader YS, Abu Slaih A, Iblan I. Blood pressure of Jordanian workers chronically exposed to noise in industrial plants. Int J Occup Environ Med. 2017:8:217-23. https://doi.org/10.15171/ijoem.201 7.1134.

34. Dimakopoulou K, Koutentakis K, Papageorgiou I, Kasdagli M-I, Haralabidis AS, Sourtzi $P$, et al. Is aircraft noise exposure associated with cardiovascular disease and hypertension? Results from a cohort study in Athens, Greece. Occup Environ Med. 2017;74:830-7. https://doi.org/10.1136/oemed-2016-1 04180.

35. Tomei G, Sancini A, Tomei F, Vitarelli A, Andreozzi G, Rinaldi G, et al. Prevalence of systemic arterial hypertension, electrocardiogram abnormalities, and noise-induced hearing loss in agricultural workers. Arch Environ Occup Health. 2013;68(4):196-203. https://doi.org/10.1080/1933 8244.2012.701245.

36. Yu Y, Paul K, Arah OA, Mayeda ER, Wu J, Lee E, et al. Air pollution, noise exposure, and metabolic syndrome - a cohort study in elderly MexicanAmericans in Sacramento area. Environ Int. 2020;134:105269. https://doi. org/10.1016/j.envint.2019.105269.

37. Jorgensen JT, Brauner EV, Backalarz C, Laursen JE, Pedersen TH, Jensen SS, et al. Long-term exposure to road traffic noise and incidence of diabetes in the danish nurse cohort. Environ Health Perspect. 2019;127:57006. https:// doi.org/10.1289/EHP4389.

\section{Publisher's Note}

Springer Nature remains neutral with regard to jurisdictional claims in published maps and institutional affiliations. 